\begin{tabular}{|c|c|c|c|}
\hline \multirow{3}{*}{$\begin{array}{r}\text { Case Reports in } \\
\text { Gastroenterology }\end{array}$} & \multicolumn{2}{|c|}{ Case Rep Gastroenterol 2020;14:56-62 } & \multirow{3}{*}{$\begin{array}{l}\text { Karger } \\
\text { Open'access }\end{array}$} \\
\hline & $\begin{array}{l}\text { DOI: } 10.1159 / 000505513 \\
\text { Published online: January 29, } 2020\end{array}$ & $\begin{array}{l}\text { () } 2020 \text { The Author(s) } \\
\text { Published by S. Karger AG, Basel } \\
\text { www.karger.com/crg }\end{array}$ & \\
\hline & $\begin{array}{l}\text { This article is licensed under the } \\
\text { International License (CC BY-NC) } \\
\text { Usage and distribution for commer }\end{array}$ & $\begin{array}{l}\text { nons Attribution-NonCommercia } \\
\text { ger.com/Services/OpenAccessLic } \\
\text { uires written permission. }\end{array}$ & \\
\hline
\end{tabular}

\title{
Hepatic Epithelioid Hemangioendothelioma: Difficult Differential Diagnosis from Angiosarcoma
}

\author{
Tomohiko Taniai $^{a}$ Shinji Onda ${ }^{a}$ Shun Sato $^{b}$ Hiroaki Shiba $^{a}$ \\ Taro Sakamoto $^{a}$ Katsuhiko Yanaga ${ }^{a}$ \\ aDepartment of Surgery, The Jikei University School of Medicine, Tokyo, Japan; \\ ${ }^{b}$ Department of Pathology, The Jikei University School of Medicine, Tokyo, Japan
}

\section{Keywords}

Epithelioid hemangioendothelioma - Differential diagnosis - Repeat hepatectomy ·

Angiosarcoma · CAMTA1

\begin{abstract}
Hepatic epithelioid hemangioendothelioma (EHE) is extremely rare, and preoperative diagnosis is difficult because hepatic EHE has clinicopathological features that are similar to those of angiosarcoma. However, it is important to differentiate hepatic EHE from angiosarcoma because the latter is an aggressive tumor with poor prognosis. We herein report a case of hepatic EHE that was difficult to distinguish from angiosarcoma by tumor biopsy. A 30-year-old man with Crohn's disease presented with multiple liver tumors. The tumors were preoperatively diagnosed as angiosarcoma by tumor biopsy. The patient underwent extended left hemihepatectomy with biliary reconstruction and partial resection of segments 6 and 8. Immunohistochemical staining was positive for CD34, factor VIII, and calmodulin binding transcription activator 1 (CAMTA1), and the pathological diagnosis was EHE. Two years after surgery, a recurrent tumor was found in liver segment 6 , for which laparoscopic partial hepatectomy was performed. Pathological examination revealed recurrence of EHE. The patient remained well with no evidence of tumor recurrence as of 9 months after the second resection. In conclusion, we
\end{abstract}




\section{Case Reports in Gastroenterology}

Case Rep Gastroenterol 2020;14:56-62

DOI: $10.1159 / 000505513$

(c) 2020 The Author(s). Published by S. Karger AG, Basel www.karger.com/crg

Taniai et al.: Hepatic Epithelioid Hemangioendothelioma: Difficult Differential Diagnosis from Angiosarcoma

described a case of hepatic EHE that was initially diagnosed as angiosarcoma on biopsy. Immunohistochemical staining with CAMTA1 may help distinguish EHE from angiosarcoma.

(C) 2020 The Author(s)

Published by S. Karger AG, Basel

\section{Introduction}

Epithelioid hemangioendothelioma (EHE) is a rare borderline malignancy that can occur in soft tissues, bones, lungs, and the liver [1]. The incidence of hepatic EHE is $<0.1$ per 100,000 population [2]. Most patients with hepatic EHE present with disseminated disease, with involvement of both lobes [3]. Hepatic EHE has clinicopathological features that are similar to those of angiosarcoma. For that reason, hepatic EHE is sometimes misdiagnosed as angiosarcoma [4]. However, it is important to differentiate hepatic EHE from angiosarcoma because the latter is an aggressive tumor with poor prognosis. We herein report a case of hepatic EHE that was difficult to distinguish from angiosarcoma by tumor biopsy.

\section{Case Report}

A 30-year-old man with Crohn's disease had undergone small bowel resection at the age of 29 years and had been stable on mesalazine 3,000 mg/day. He had not been exposed to polyvinyl chloride. Computed tomography (CT) revealed a low-density area in segment 8 of the liver; an inflammatory pseudotumor was suspected and was followed periodically. At the age of 32 years, abdominal enhanced CT revealed multiple low-density tumors in both lobes of the liver with ring enhancement in the early and portal phases (Fig. 1a, b). Most of the lesions were located in the liver surface. The intrahepatic bile duct in segment 4 was dilated (Fig. 1a). Magnetic resonance imaging (MRI) with contrast enhancement showed ring-enhanced tumors in both lobes of the liver, which were defective in hepatocyte phase (Fig. 1c, d), with the dilation of the intrahepatic duct in segment 4 (Fig. 1e). Abdominal ultrasound with contrast enhancement revealed an early ring-enhanced tumor in segment 8 , and its central lesion was completely defective in the postvascular phase (Fig. 1f). Other tumors also showed similar enhancement. Laboratory data showed hemoglobin $12.6 \mathrm{~g} / \mathrm{dL}$, alkaline phosphatase $348 \mathrm{U} / \mathrm{L}$, gamma-glutamyltranspeptidase $80 \mathrm{U} / \mathrm{L}$, and albumin $3.4 \mathrm{~g} / \mathrm{dL}$. Serum levels of tumor markers, including alpha-fetoprotein and protein induced by vitamin $\mathrm{K}$ absence or antagonist-II were normal. Hepatitis B and C viral markers were negative. Percutaneous needle biopsy revealed mild to severe dysplasia and invasive growth. Immunohistochemical staining was positive for CD34 and factor VIII. The tumors were preoperatively diagnosed as angiosarcoma for which extended left hemihepatectomy with biliary reconstruction because of hilar invasion that was diagnosed during surgery and partial resection of segments 6 and 8 were performed. Macroscopic examination of the resected specimen revealed multifocal tumors (Fig. 2a, b). Pathologically, the tumors comprised moderate to severe sclerosis, moderate cell density, and mild to severe dysplasia (Fig. 2c). Immunohistochemical staining was positive for CD34 (Fig. 2d), factor VIII (Fig. 2e), and calmodulin binding transcription activator 1 (CAMTA1). The pathological diagnosis was EHE (Fig. 2c). The patient made a satisfactory recovery and was discharged on postoperative day 16 . Two years after surgery, a 1.6-cm tumor was found on the surface of liver segment 6 on CT and MRI. CT revealed a low-density tumor without ring enhancement. MRI with contrast enhancement showed a low-intensity tumor, which was defective in hepatocyte phase. The tumor was diagnosed as recurrent EHE, for 
Taniai et al.: Hepatic Epithelioid Hemangioendothelioma: Difficult Differential Diagnosis from Angiosarcoma

which laparoscopic partial hepatectomy was performed (Fig. 3a). Pathological findings were similar to those of the first operation (Fig. 3b), and immunohistochemical staining was positive for CD31 (Fig. 3c) and CD34 (Fig. 3d), which was compatible with recurrent EHE. The patient remains well, with no evidence of tumor recurrence as of 9 months after the second resection.

\section{Discussion/Conclusion}

Three-fourths of patients with hepatic EHE are initially misdiagnosed [5]. The differential diagnosis includes angiosarcoma, cholangiocarcinoma, hepatocellular carcinoma, metastatic carcinoma, sclerosing hemangioma, and inflammatory pseudotumor, among others [5]. The differential diagnosis is difficult, especially between hepatic EHE and angiosarcoma. The distinguishing features are as follows. Hepatic EHE has a predilection for age 40-50 years, while angiosarcoma has a predilection for age 60-80 years. Symptoms of hepatic EHE and angiosarcoma are very similar: abdominal pain, weight loss, weakness, anorexia, and asymptomatic [3, 6]. Imaging studies sometimes reveal similar findings between hepatic EHE and angiosarcoma. Hepatic EHE showed three patterns in contrast-enhanced imaging: peripheral nodular enhancement with centripetal fill-in and wash-out during the portal venous and late venous phase (PVLP), rim-like arterial enhancement with wash-out during the PVLP, and inversed target sign with/without wash-out during the PVLP [7]. Moreover, hepatic EHE tends to be multiple and located in the periphery [3]. Angiosarcoma presents differently on CT imaging according to its various morphological patterns which depend on its necrosis and hemorrhage [6]. Pathologically, hepatic EHE tends to show preserved hepatic acinar structure, severe sclerosis, moderate cell density, and mild dysplasia. In contrast, angiosarcoma tends to show destroyed hepatic acinar structure, mild sclerosis, high cell density, and severe dysplasia. Immunohistochemical staining of CD31, CD34, and Factor VIII is positive in both hepatic EHE and angiosarcoma. In the current case, the tumor biopsy specimen showed mild to severe dysplasia and was positive for CD34 and Factor VIII, both of which are found in angiosarcoma and hepatic EHE. Hepatic EHE and angiosarcoma are known to have many similar clinicopathological features.

Approximately $90 \%$ of EHE patients have a WW domain containing transcription regulator 1-calmodulin binding transcription activator 1 (WWTR1-CAMTA1) fusion gene and show overexpression of CAMTA1 [8]. CAMTA1 is associated with memory performance, which is normally detectable in brain neurons [9]. The frequency of the expression of CAMTA1 in other epithelioid mesenchymal tumors was reported as almost $0 \%$ [8]. In particular, the frequency of the expression of CAMTA1 in angiosarcoma was only 4\% [8]. Therefore, immunohistochemical staining with CAMTA1 is more useful for diagnosis of EHE. However, staining with CAMTA1 was not performed in our hospital. In the current case, staining with CAMTA1 was only performed in the postoperative specimen as a consignment. CAMTA1 immunohistochemical staining of a liver biopsy specimen may be helpful for preoperative differential diagnosis of EHE and angiosarcoma, and we suggest that staining with CAMTA1 should be more popular to make diagnosis of EHE.

In patients with hepatic EHE, liver resection and transplantation are associated with better outcomes, as compared to those treated with chemotherapy and radiotherapy [3]. The 5year survival rate of all patients with hepatic EHE is $41.1 \%$, while that after liver resection is $75 \%$ [3]. Therefore, liver resection is recommended if possible. In patients with hepatic angiosarcoma, liver resection is also recommended as initial treatment [10]. The median overall 
survival of all patients with hepatic angiosarcoma is 5 months, whereas the median survival of patients after liver resection or in combination with adjuvant therapy is 17 months [11]. Therefore, liver resection is recommended as initial treatment both in hepatic EHE and angiosarcoma, although prognosis after resection is different between them. The current case remained well at 16 months after the first operation, but long-term follow-up is needed to clarify the role of surgery for hepatic EHE. In conclusion, we described a case of hepatic EHE that was preoperatively diagnosed as angiosarcoma on needle biopsy. The differential diagnosis of hepatic EHE and angiosarcoma is difficult, but immunohistochemical staining with CAMTA1 may be helpful.

\section{Statement of Ethics}

Written informed consent was obtained from the patients for publication of this case report and any accompanying images.

\section{Disclosure Statement}

The authors have no conflicts of interest to declare.

\section{Funding Sources}

None.

\section{Author Contributions}

T.T., S.O., S.S., H.S., T.S. drafted the manuscript. S.S. advised other authors about pathological findings and made immunohistochemical staining for figures. K.Y. has given the final approval of the version to be published. All authors read and approved the final manuscript.

\section{References}

1 Mehrabi A, Hoffmann K, Weiss KH, Mogler C, Schemmer P, Büchler MW, et al. Long term follow up after resection emphasizes the role of surgery in Primary Hepatic Epithelioid Hemangioendothelioma. Ann Med Surg (Lond). 2016 Mar;11:1-4.

2 Hertl M, Cosimi AB. Liver transplantation for malignancy. Oncologist. 2005 Apr;10(4):269-81.

3 Mehrabi A, Kashfi A, Fonouni H, Schemmer P, Schmied BM, Hallscheidt P, et al. Primary malignant hepatic epithelioid hemangioendothelioma: a comprehensive review of the literature with emphasis on the surgical therapy. Cancer. 2006 Nov;107(9):2108-21.

4 van Rosmalen BV, Verheij J, Phoa SSKS, van Gulik TM. Hepatic epithelioid haemangioendothelioma (HEHE): a diagnostic dilemma between haemangioma and angiosarcoma. BMJ Case Rep. 2017 Nov;2017:bcr2017220687.

5 Makhlouf HR, Ishak KG, Goodman ZD. Epithelioid hemangioendothelioma of the liver: a clinicopathologic study of 137 cases. Cancer. 1999 Feb;85(3):562-82.

6 Zhu YP, Chen YM, Matro E, Chen RB, Jiang ZN, Mou YP, et al. Primary hepatic angiosarcoma: A report of two cases and literature review. World J Gastroenterol. 2015 May;21(19):6088-96. 


\begin{tabular}{l|l}
\hline Case Rep Gastroenterol 2020;14:56-62 \\
\hline DOI: 10.1159/000505513 & $\begin{array}{l}\text { @ 2020 The Author(s). Published by S. Karger AG, Basel } \\
\text { www.karger.com/crg }\end{array}$ \\
\hline
\end{tabular}

Taniai et al.: Hepatic Epithelioid Hemangioendothelioma: Difficult Differential Diagnosis from Angiosarcoma

7 Klinger C, Stuckmann G, Dietrich CF, Berzigotti A, Horger MS, Debove I, et al. Contrast-enhanced imaging in hepatic epithelioid hemangioendothelioma: retrospective study of 10 patients. Z Gastroenterol. 2019 Jun;57(6):753-66.

8 Doyle LA, Fletcher CD, Hornick JL. Nuclear expression of CAMTA1 distinguishes epithelioid hemangioendothelioma from histologic mimics. Am J Surg Pathol. 2016 Jan;40(1):94-102.

9 Huentelman MJ, Papassotiropoulos A, Craig DW, Hoerndli FJ, Pearson JV, Huynh KD, et al. Calmodulinbinding transcription activator 1 (CAMTA1) alleles predispose human episodic memory performance. Hum Mol Genet. 2007 Jun;16(12):1469-77.

10 Weitz J, Klimstra DS, Cymes K, Jarnagin WR, D’Angelica M, La Quaglia MP, et al. Management of primary liver sarcomas. Cancer. 2007 Apr;109(7):1391-6.

11 Zheng YW, Zhang XW, Zhang JL, Hui ZZ, Du WJ, Li RM, et al. Primary hepatic angiosarcoma and potential treatment options. J Gastroenterol Hepatol. 2014 May;29(5):906-11.
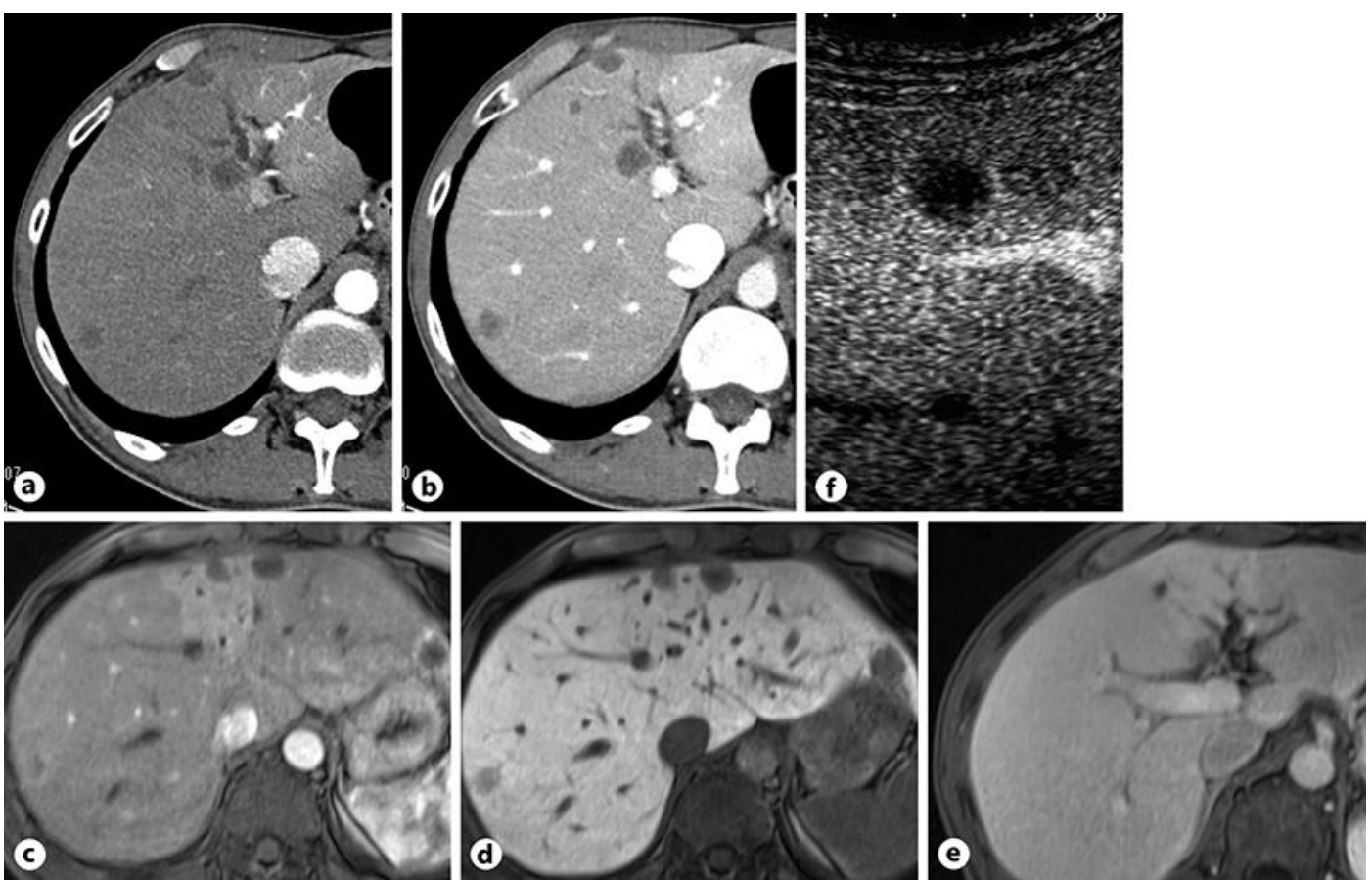

Fig. 1. Computed tomography revealed low density tumors in both lobes of the liver (a) with ring enhancement in portal phase (b). Intrahepatic bile duct in segment 4 was dilated. Gadoxetic acid-enhanced magnetic resonance imaging identified low-intensity tumors in both lobes of the liver with ring enhancement (c), which were defective in the hepatocyte phase (d). e MRI also revealed the dilation of intrahepatic duct in segment 4. f Abdominal ultrasound with contrast enhancement showed an early ring-enhanced tumor, and its central lesion was completely defective in the postvascular phase. 


\section{Case Reports in Gastroenterology}

\begin{tabular}{l|l}
\hline Case Rep Gastroenterol 2020;14:56-62 \\
\hline DOI: 10.1159/000505513 & $\begin{array}{l}\text { @ 2020 The Author(s). Published by S. Karger AG, Basel } \\
\text { www.karger.com/crg }\end{array}$ \\
\hline
\end{tabular}

Taniai et al.: Hepatic Epithelioid Hemangioendothelioma: Difficult Differential Diagnosis from Angiosarcoma
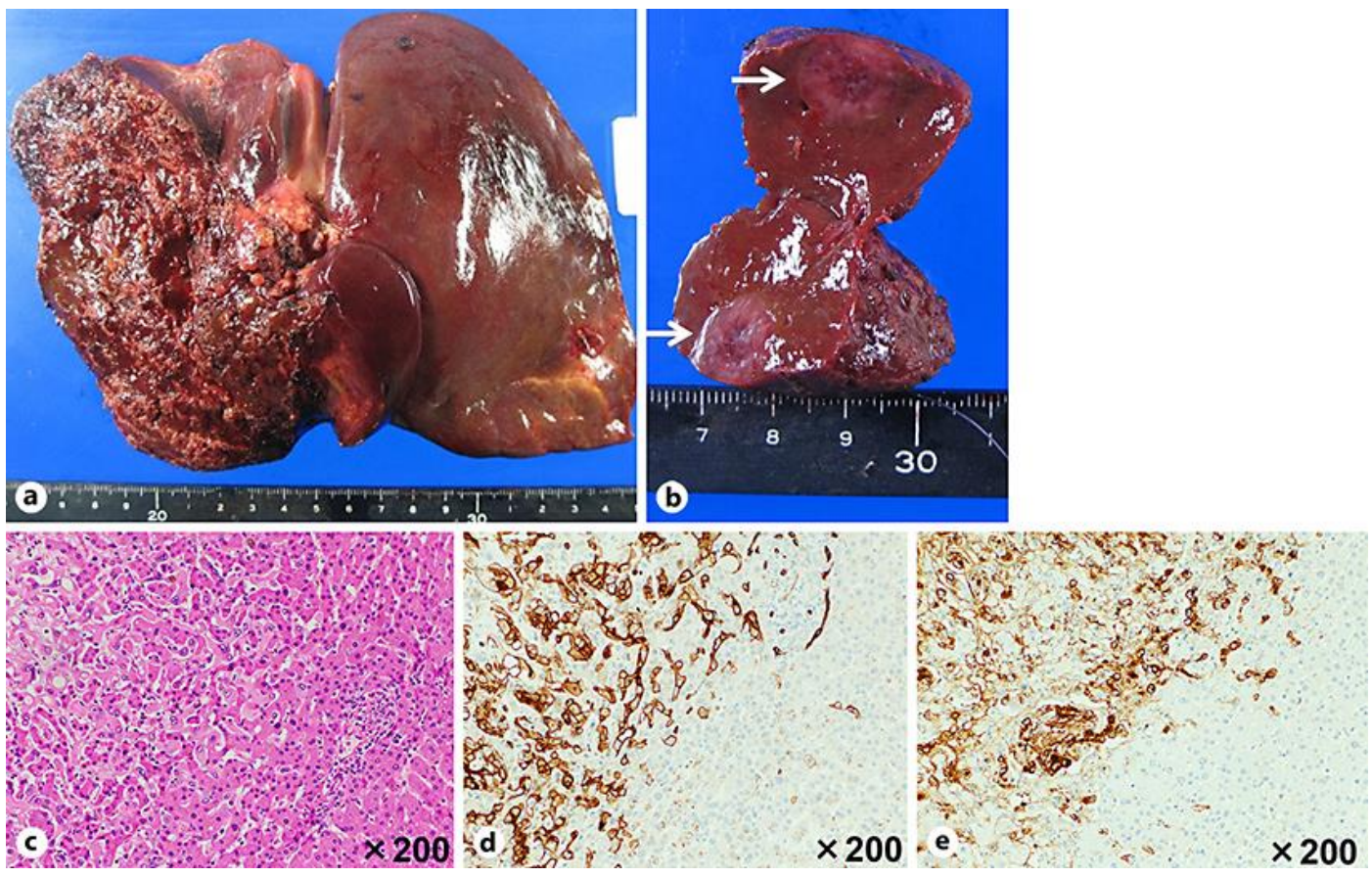

Fig. 2. The resected liver specimen (a, b) showed multifocal tumors (b, arrows). Immunohistochemical staining was positive for CD34 (d, left side) and factor VIII (e, left side), and pathological examination revealed epithelioid hemangioendothelioma (c, left side). Normal liver parenchyma (c, right side) showed negative staining for CD34 (d, right side) and factor VIII (e, right side). 


\section{Case Reports in Gastroenterology}

\begin{tabular}{l|l}
\hline Case Rep Gastroenterol 2020;14:56-62 \\
\hline DOI: 10.1159/000505513 & $\begin{array}{l}\text { ○ 2020 The Author(s). Published by S. Karger AG, Basel } \\
\text { www.karger.com/crg }\end{array}$ \\
\hline
\end{tabular}

Taniai et al.: Hepatic Epithelioid Hemangioendothelioma: Difficult Differential Diagnosis from Angiosarcoma

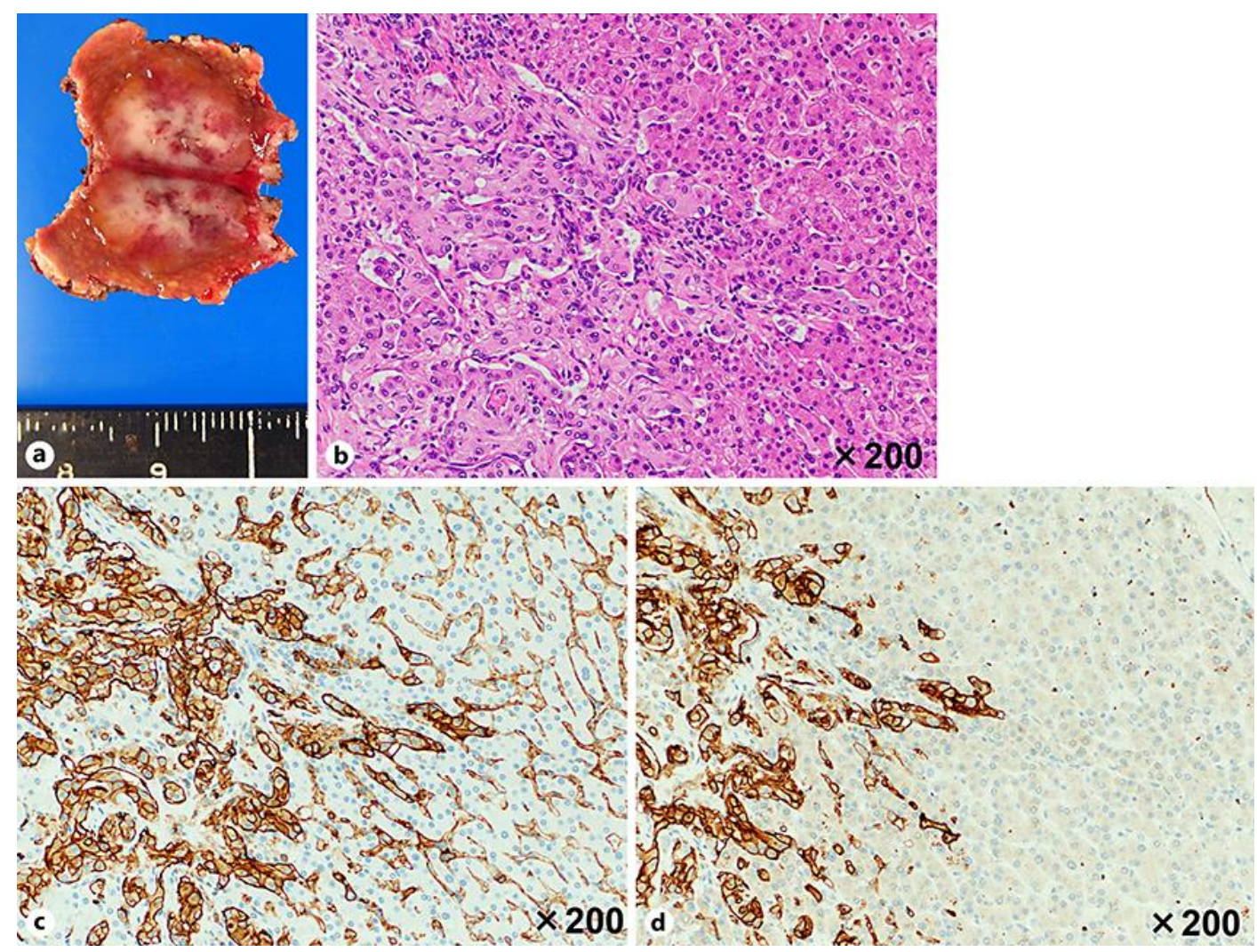

Fig. 3. a Resected liver specimen. Immunohistochemical staining was positive for CD31 (c, left side) and CD34 (d, left side). Pathological examination revealed recurrence of epithelioid hemangioendothelioma (b, left side). Normal liver parenchyma (b, right side) revealed negative staining for CD31 (c, right side) and CD34 (d, right side). Usually, normal sinusoidal endothelium cells were positive for CD31 (c, right side). 